\title{
LASER TRIMMING OF THICK FILM RESISTORS
}

\author{
A. F. DYSON and A. J. CABLE \\ Erie Electronics Ltd., Gt. Yarmouth, Norfolk, U.K. \\ (Received October, 1973; in final form January 21, 1974)
}

\begin{abstract}
Recent papers and conferences, e.g. Ref. 1, have indicated very strongly that thick film screen processing has become a routine production item, with the specifications and economics being considered in detail. ${ }^{2}$ The existing resistor and conduction materials and processes have become well documented ${ }^{3,4}$ and are well known on an international scale. This paper reviews the present situation and, in particular, discusses the effects of a recently-introduced production tool - the laser trimmer.
\end{abstract}

\section{INTRODUCTION}

Thick film technology refers to the science of laying down by screen printing techniques conductors, resistors and capacitors, - the screen printable material being in the form of a thixotropic paste. The materials generally used for the conductors include platinum/gold, palladium/gold and palladium/silver. ${ }^{5}$ The precious metal, in a finely divided form, together with a suitable glass frit which is generally a borosilicate glass with the addition of the oxides of zinc and/or magnesium, are introduced into a carrier, typically ethyl cellulose, together with a solvent dibutyl carbitol acetate.

Resistor materials currently in general use are divided into two categories, palladium oxide/silver systems and the more recent ruthenium oxide ${ }^{6}$ system. Again the basic metal and metal oxide, in a finely divided form, is mixed with glass frit,carrier and solvent system, - turpineol being a preferred solvent.

Screen printed capacitors are generally composed of finely divided particles of a pre-fired barium titanate dielectric, again contained in a glass frit with an organic carrier. The existing materials used for screen printing capacitors have showed certain disadvantages, particularly with regard to porosity which causes pinholes in the dielectric layer and poor humidity performance. A recent paper ${ }^{7}$ has introduced a modification of this type of material which shows the possibilities of improvement of these deficiencies.

The conductor, resistor and capacitor materials appear as manufactured in a thick paste form with typical viscosities of $150,000 \mathrm{cps}$. It is important from the production viewpoint that the materials are held at constant temperature, $\pm 2^{\circ} \mathrm{C}$, and remain homogenous, generally achieved by slow stirring. The screening process is a modern adaptation of the silk screen printing process commonly used for display printing of posters, etc. However, as will be well appreciated, great demands are made of the resulting print in terms of dimensional accuracy. Very sophisticated thick film printing machines are available which automatically enable close mechanical tolerances to be maintained. The screen itself is generally made of stainless steel mesh, having typical wire diameter of $0.002^{\prime \prime}$ and 200 wires to the linear inch, giving an effective open area of $36 \%$. The pattern is laid down by photolithographical techniques. The materials are squeezed through the pattern by a pneumatically operated paddle which is automatically controlled in speed and pressure. These materials, after screening, are dried and then fired through a closely temperature controlled profile furnace with firing temperatures ranging between $750^{\circ} \mathrm{C}$ and $950^{\circ} \mathrm{C}$. The resulting thick film has a thickness of approximately 0.0005 inches to 0.0012 inches.

The substrate onto which the thick films are screened is a high purity alumina, the general grade being $96 \%$ alumina.

The techniques involved in laying down these films and the subsequent processing have now been developed so that it has become a routine production process on an international scale with a great commonality between the various companies involved in the methods used to obtain satisfactory products. Most of the work presently going on is aimed at the subsequent processing stages which includes the trimming operation, in which process the resistor is adjusted from its fired value to the exact value required. Also of importance are the methods 
involved in turning such a thick film passive circuit into a hybrid circuit, which involves the attachment of active and passive devices and the subsequent encapsulation processes. (See the papers given on attachment at the Hybrid Microelectronics Conference at Canterbury. ${ }^{1}$ )

It is not intended in this paper to consider approaches being made in the attachment and encapsulation processes. We are interested in examining the present state of the trimming of the resistors, - in this case by the use of an automatic laser trimming system.

Although laser trimming was introduced in the U.S.A. some years ago, it is only in the last two years that the modern, computer-controlled, fully automatic equipments have been introduced to large scale production. It can be said at this point that the introduction of such a revolutionary technique has proceeded with far less problems than might have been expected. Although there is still some more work to be done in this field, it is useful to consider the problems as they are presently known and to suggest some methods of overcoming them.

\section{THICK FILM RESISTOR ADJUSTING}

The final fired value of a screened resistor depends on many variables, the more major of which are:-

1) Effective resistor geometry.

2) Resistor film thickness.

3) Final fired composition of film.

Items 1 and 2 are dependent on mechanical considerations. Item 3 is dependent on the chemical nature of the resistive material and the effects on this material of the firing process, particularly with regard to peak temperature, firing furnace profile and firing furnace atmospheres.

The resistive paste is available in a number of basic resistivities which are specified in ohms per square. A typical range would comprise $50 \mathrm{ohms}, 100 \mathrm{ohms}$, $500 \mathrm{ohms}, 1,000 \mathrm{ohms}, 10,000 \mathrm{ohms}, 50,000 \mathrm{ohms}$, and $100,000 \mathrm{ohms}$. These are generally designated as the primary range. The user blends selected primaries together to obtain the specified value paste for a given resistive circuit.

The early palladium oxide/silver systems were particularly sensitive to all furnace conditions and, as a result, fired value variations of $\pm 25 \%$ were common and accepted. To obtain the right value of resistor it was therefore necessary to design the resistor film, after firing, to lie in the range $+0 \%-50 \%$ of nominal value. By making cuts into the fired resistor film, the final values at the required tolerance could be obtained. The more recent introduction of ruthenium oxide resistive materials has reduced the spread of fired resistor values, due to the fact that the material is very much less sensitive to firing furnace conditions. Typical spreads of $12 \%$ are obtained. However, resistor adjusting will still be required to obtain close tolerance resistors.

The theory of thick film resistor cutting is easily explained. Figure 1a shows a thick film resistor. If the fired value of the film is $100 \Omega$ per square and $\mathrm{A}=\mathrm{B}$ then the resistor value will be $100 \Omega$.

Figure 1b shows a similar resistor as in Figure 1a, but resistor film has been removed in the area shown as slot $\mathrm{C}$. If $\mathrm{D}=1 / 2 \mathrm{~A}$, then the resistance of area $\mathrm{E}$ will be $50 \Omega$ and this will also be the case for area F. The bridging resistor $\mathrm{YxY}$ will also have a value of $50 \Omega$ if $X=1 / 2 Y$. However, the resistance between the terminals $\mathrm{Z}$ and $\mathrm{V}$ will not be the simple sum of these three values because current will not be distributed evenly throughout areas $\mathrm{E}$ and $\mathrm{F}$. A precise answer to this problem can be obtained using a curvilinear square analysis, but an approximate solution can be arrived at by assuming that current in area $E$ is contained in the triangular region bounded by the top uncut side, the electrode contact and a line joining

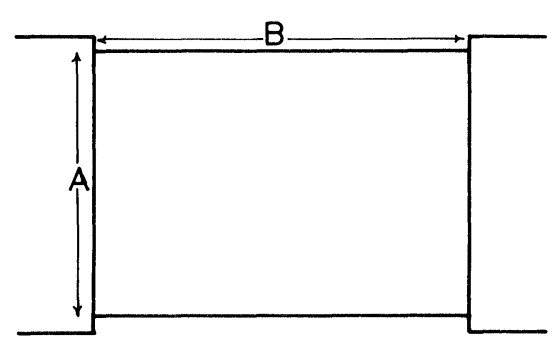

FIGURE 1 (a) Thick film resistor.

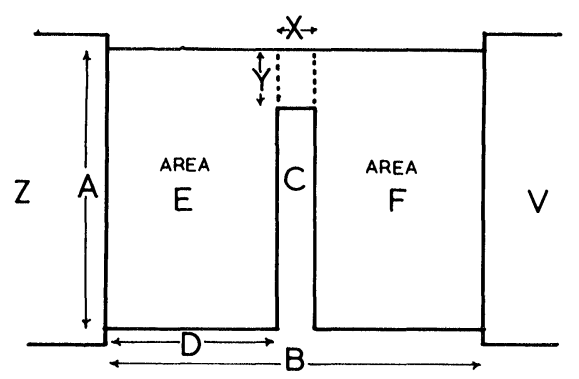

FIGURE 1 (b) Thick film resistor after adjustment. 
the bottom contact point of the electrode $\mathrm{Z}$, to the top of the cut nearest to electrode $\mathrm{Z}$.

With such an approximate analysis, and given $\mathrm{X}=\mathrm{Y}$, the value of the resistance of area $\mathrm{E}$ will be $235 \Omega$. If the cut width is $1 / 100^{\text {th }}$ of the length of the resistor $\mathrm{B}$, this will mean that for such a configuration the total resistance would be $235+100+235 \Omega$, i.e. $\sim 570 \Omega$.

This simple example illustrates an approach to thick film resistor adjusting which is also known as resistor abrading or resistor cutting. However, the limiting factor in designing such a resistor for adjustment is one of power dissipation. Each resistive paste manufacturer specifies for each material family suggested power loadings, generally expressed in watts per square inch. In addition, information on maximum film temperature and the effects of the thermal resistance of various substrates is also given.

For a paste that will give a film that will withstand 20 watt per square inch, 20 watts will be the dissipation allowed in the resistor represented by Figure 1a if $\mathrm{A}=\mathrm{B}=1^{\prime \prime}$. With the configuration shown in Figure $1 \mathrm{~b}$ however, the maximum dissipation will

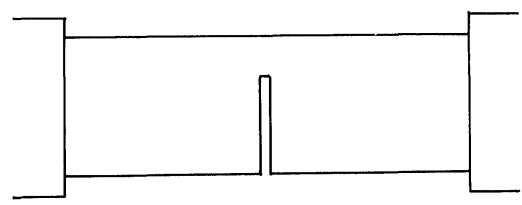

(a) Single cut.

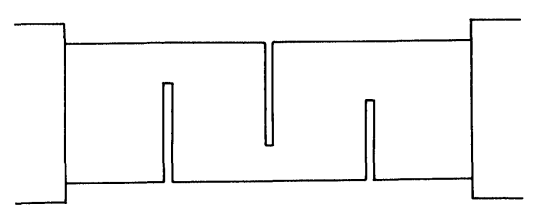

(c) Serpentine cut.

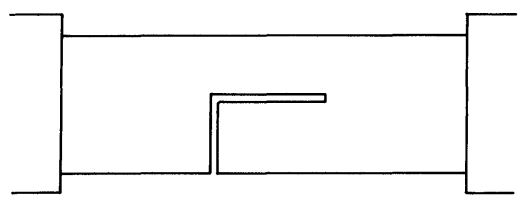

(e) L-cut. be set by the current carrying capabilities of the bridge section $\mathrm{XY}$. If resistor $\mathrm{XY}$ is $0.1^{\prime \prime} \times 0.1^{\prime \prime}$ i.e. 0.01 square inches, then this section has an allowed dissipation of 0.2 watts and this will provide the limit of the total resistor.

Figure $1 \mathrm{a}$ and $1 \mathrm{~b}$ have illustrated an extreme case and in practice, this single slot cutting would not be used on this resistor. Figures 2 (a) to (f) show various types of cut which have been and are being used to adjust thick film resistors.

\section{ADJUSTING METHODS}

\section{Air Abrading}

The original method of removing thick film material to increase the resistance value to the nominal value required, used so-called 'air-abrasive' techniques, - in simple terms, sand blasting. A jet of dolomite powder with a typical grain size of 40 micron is forced through a nozzle, having a typical diameter of $0.007^{\prime \prime}$, by compressed gas. The nozzle is controlled and

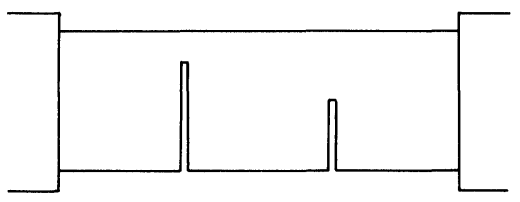

(b) Double cut.

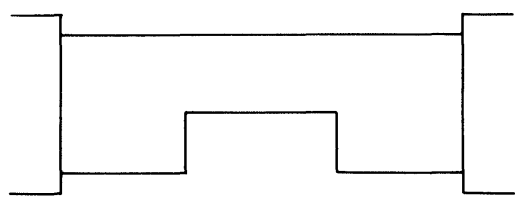

(d) Broad front cut.

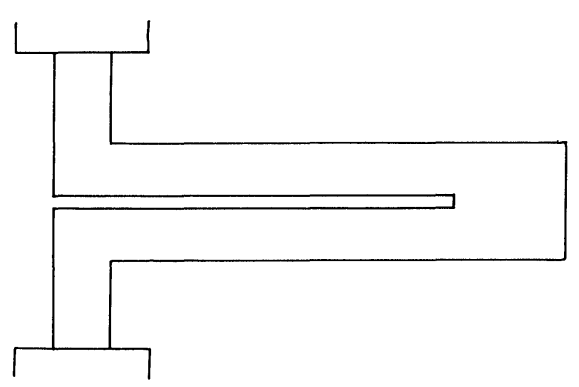

(f) Top hat cut.

FIGURE 2 Typical cuts used for thick film resistors. 
moveable in the $\mathrm{x}$ and $\mathrm{y}$ planes but is fixed in height, the nozzle orifice being typically $0.025^{\prime \prime}$ from the substrate. As the resistor material is removed, the resistance value changes, and this information is monitored by a measurement unit and the nozzle continues to move and cut until the set resistance value is achieved.

This technique has the following advantages:

a) The equipment is relatively cheap.

b) The cut is made with little damage to the remaining resistor material.

and the following disadvantages:

a) Relatively slow.

b) Difficult to cut more than four resistors on a substrate in one pass due to the physical size of the nozzle.

When large production volumes are required, it is necessary to consider more sophisticated equipments.

\section{Laser Adjusting}

Laser adjustment of both thin and thick film resistors has been used for some considerable time. However, it is only in latter years that very high speed automatic equipments have come into worldwide use. ${ }^{8}$ The way in which the laser is operated dictates the degree of success of the method to a very large extent. At present the type of laser almost universally used in YAG:Nd (Yittrium Aluminium Garnet doped with Neodymium) with a wavelength around 1.06 microns. Early laser systems, utilising $\mathrm{CO}_{2}$ with wavelengths of 10.6 microns, appeared to dissipate the energy more in the substrate than in the resistive material. The YAG:Nd laser wavelength appears such that a large part of the dissipation is in the resistive material, this is probably due to the shorter wavelength permitting a smaller focussed beam diameter.

Given the type of laser, there are still many variables to be decided before good trimming results can be consistently obtained and an appreciation of these variables and their effects is gained only from an understanding of the cutting method, coupled with painstaking testing of the finished product.

The laser cuts a channel in the resistive material by vapourising a series of overlapping holes, allowing time in between for a very fast electronic bridge to measure the resistor value and thus control the beam. The distance the beam moves between holes and the actual beam diameter govern the degree of overlap; this distance is known as the 'bite size'. The number

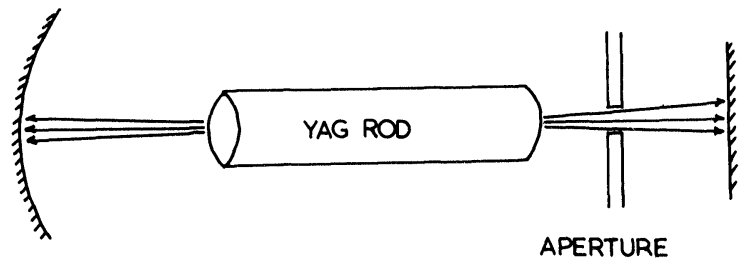

FIGURE 3 Laser action TEM $_{0_{0}}$ mode:

of holes cut per second is known at the 'q-rate' and this, together with the bite size, governs the speed at which the laser cuts. The laser pulse that vapourises the resistive material is itself only 100-200nS long and is a function of the YAG rod and so is not variable, although the peak power of that pulse which is around $1 \mathrm{KW}$, is dependent upon the q-switching frequency, having a maxima around $4-5 \mathrm{KHz}$. The laser input power can of course be varied within certain limits, thus moving this frequency-dependent relationship up and down the power scale. The laser operating mode is also a prime factor to consider, the two modes most usually specified being $\mathrm{TEM}_{00}$ and multimode. The YAG laser can be made to operate in $\mathrm{TEM}_{00}$ (Transverse Electro-Magnetic - magnetic vector zero order, electric vector zero order) by the use of a simple aperture as shown in Figure 3. The longer beam paths evident in Figure 4 are eliminated,

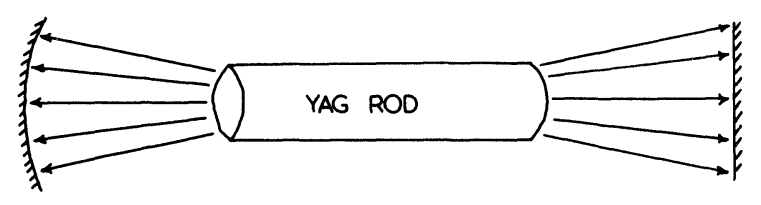

FIGURE 4 Laser action - Multimode.

thus giving a beam concentrated in the centre of the YAG rod having a very narrow frequency spectrum and excellent spatial coherence. The energy distribution across the $\mathrm{TEM}_{0_{0}}$ beam is gaussian, (Figure 5) having a peak in the centre which gives the effective small spot size required for trimming purposes. As the aperture is widened, more natural modes can occur, allowing destructive interference in the magnetic and electric vectors, for example, in $\mathrm{TEM}_{01}$ the energy distribution across the beam is as shown in Figure 6.

Figure 4 indicated that for a laser operating in multimode, the various orders of the magnetic and electric vectors are governed only by the rod itself, this gives a diffuse beam containing complicated interference patterns, the result of which is a larger spot diameter containing a relatively low power 


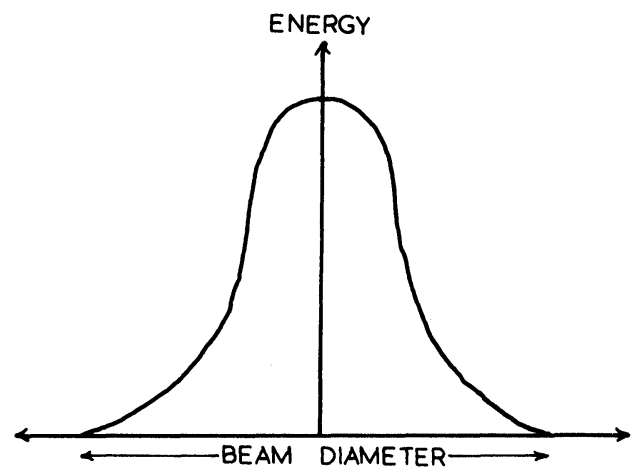

FIGURE 5 Beam energy distribution $-\mathrm{TEM}_{00}$

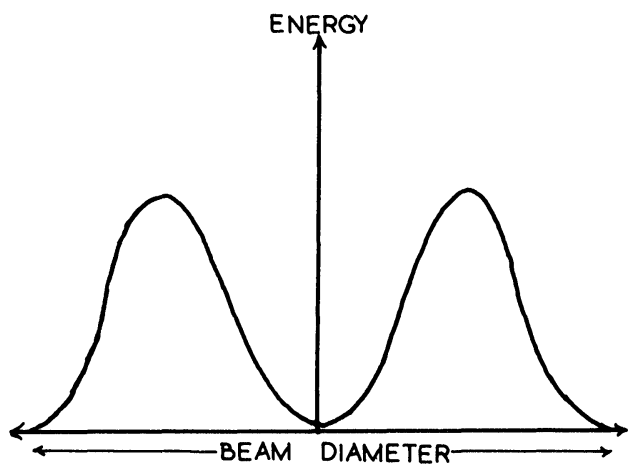

FIGURE 6 Beam energy distribution $-\mathrm{TEM}_{01}$

density. However, because the whole of the rod diameter is utilised, the overall power output is the maximum of the particular rod. Scanning electron micrographs can be obtained of cuts, using a laser in the different modes. Figure 7 shows a scanning electron micrograph of a $\mathrm{TEM}_{0_{0}}$ cut. Figure 8 shows a scanning electron micrograph of a multimode cut.

It can now be seen that there are four basic variables to consider when laser trimming, i.e., laser input power, laser mode, q-rate, and bite size. This is without considering variables such as screening quality and thickness, the type of cut used on various designs of resistor, and of course the type of resistive paste used. When faced with the task of selecting the optimum settings of the major variables, it is obviously impracticable to manipulate all four independently. The problem solves itself to a large extent if certain flexible guidelines are laid down which limit the use of the variables. The guidelines used in the present work were:

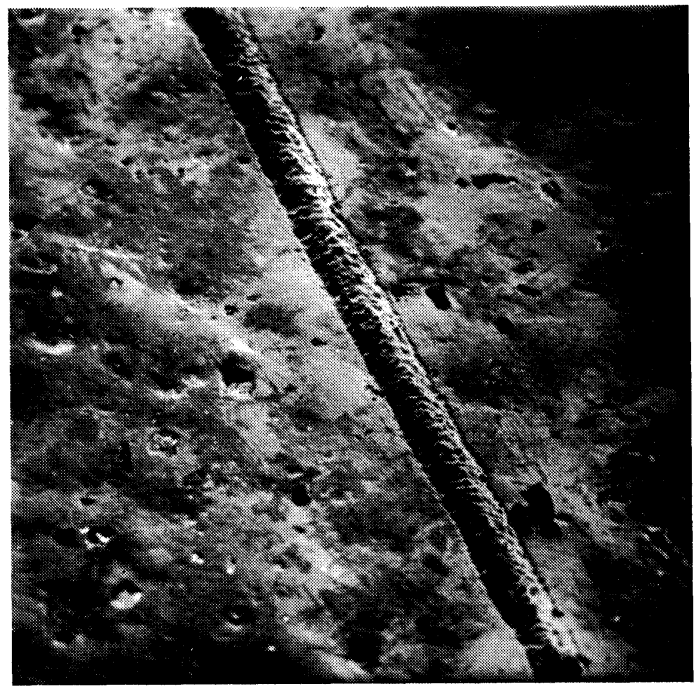

FIGURE 7 Scanning electron micrograph of cut using $\mathrm{TEM}_{00}$ mode laser beam (length of side $0.55 \mathrm{~mm}$ ) [x126].

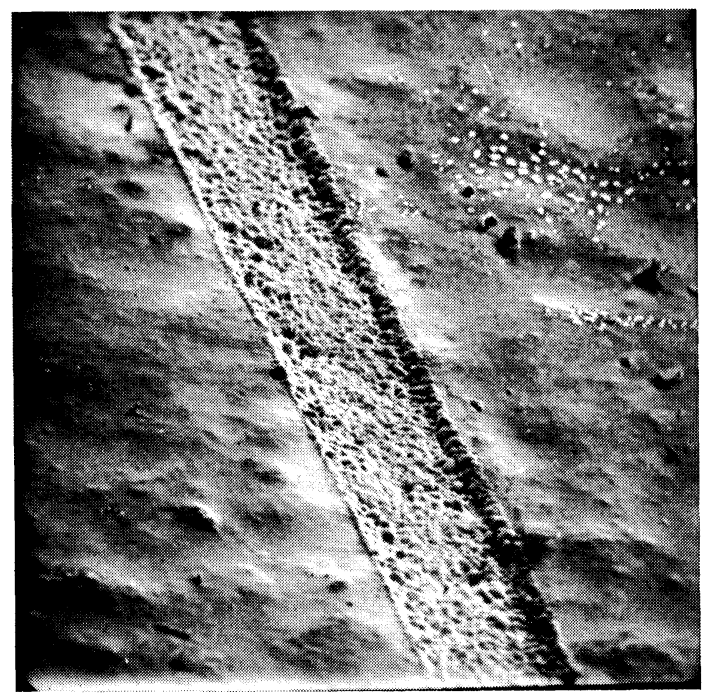

FIGURE 8 Scanning electron micrograph of cut using multimode laser beam (length of side $0.55 \mathrm{~mm}$ ) [x126] .

i) The resistors are to be trimmed to their target value with an accuracy better than $0.2 \%$.

ii) The laser cuts in the resistive material must subjectively be considered 'clean cuts'. A clean cut may be defined as the removal of all resistive material in the track to the substrate. This can be checked with a $\times 20$ microscope using back lighting on the substrate. 
Using these guidelines, we can now start to eliminate some of our variables. Number i) will limit our bite size because we cannot allow one bite of the laser to alter the resistor by more than $0.2 \%$, remembering that the automatic bridge measures only between pulse. Number ii) will limit our trimming speed thus tying down the q-rate. We then have two variables; laser mode and laser input power. To investigate the effects of these on the finished product, well over thirty batches of resistive substrates were trimmed and load tested for drift and instability.

\section{(A) A Low Value Resistor Trimmed to 20 Ohms.}

$\begin{array}{cccc}\text { Q-rate } & \text { Bite size } & \text { Laser average power } & \text { Technique } \\ 4 \mathrm{KHz} & \begin{array}{c}0.001^{\prime \prime} \\ \text { approx. }\end{array} & \text { TEM }_{0 \text { o }} 1.4 \text { watts } & \text { Double cut }\end{array}$

The change in value in these resistors between trimming and processing (involving a solder dip) varied from +1.5 to $+2.0 \%$. On examination of the resultant laser cut in the resistive material it was found that there was incomplete removal of resistive material. Subsequent attempts to ensure that all resistive material was removed from the cut proved very difficult and only very high powers and low beam speed (low bite size) enabled the complete removal of the resistive material. This very high power, low speed method immediately introduced its own problems in terms of excessive microcracking and high instabilities in the adjusted resistors.

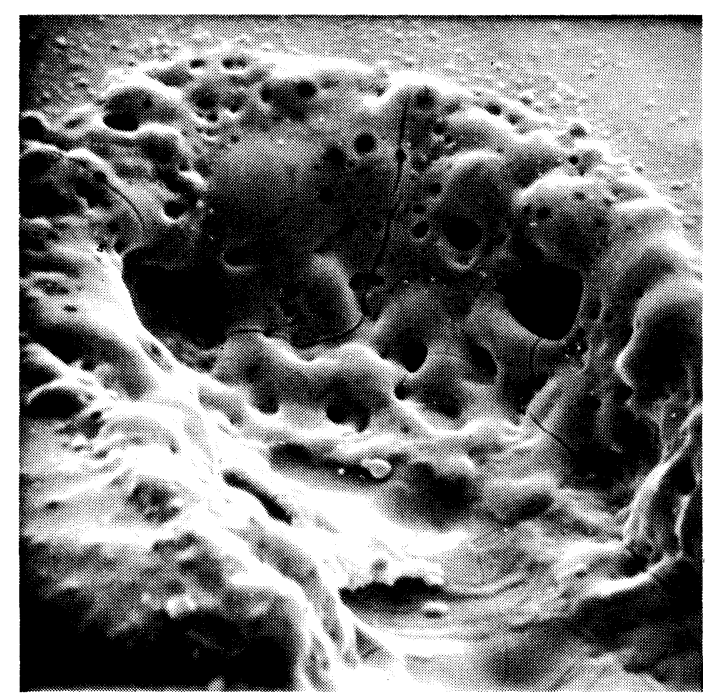

FIGURE 9 Scanning electron micrograph showing microcracking (length of side $50 \mu \mathrm{m}$ ) [ $\times 1400]$.

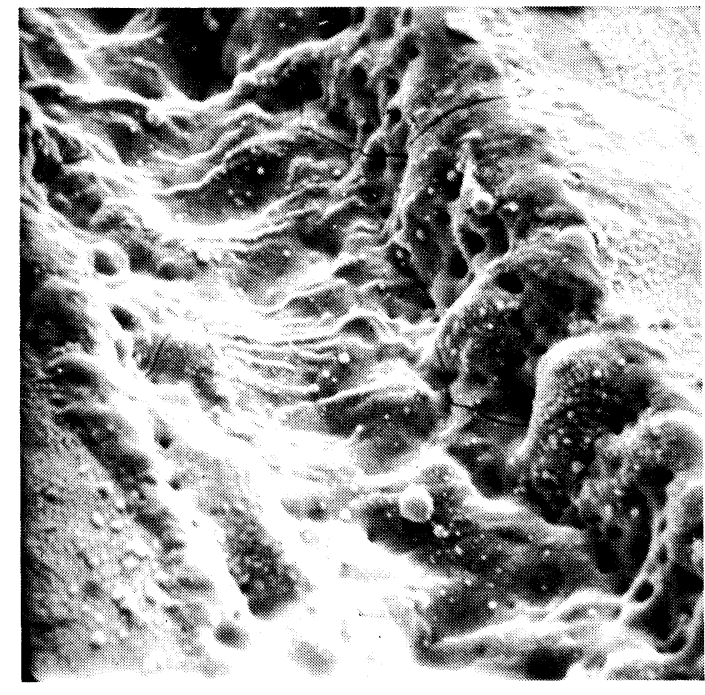

FIGURE 10 Scanning electron micrograph showing microcracking (length of side $55 \mu \mathrm{m}$ ) [x1260].

Figures 9 and 10 show micrographs of typical microcracking.

It appears that low value resistors are therefore difficult to adjust with a laser. Parallel work with air-abrasive adjusting revealed similar problems on resistor stability after adjusting on this particular material. It is therefore likely that the nature of fired resistive material itself contributes to the instabilities noted irrespective of cutting techniques.

\section{(B) Resistor Trimmed to $3.5 \mathrm{~K}$ Ohms}

$\begin{array}{llll}\text { Q-rate } & \text { Bite size } & \text { Laser average power } & \text { Technique } \\ 5 \mathrm{KHz} & 0.002^{\prime \prime} & \text { Multimode 5 watts } & \text { L-cut }\end{array}$

Test conditions

Processing (including solder dip)

100 Hrs load

$250 \mathrm{Hrs}$ load

This illustrates clearly the rapid initial drift which generally slows down with prolonged loading. The drift in resistor value experienced is unacceptably high and is attributed to the use of the laser in multimode.

The L-cut technique (Figure 2(e)) is rarely used in laser cutting because, as can be seen, a large cut is made parallel to the direction of current flow. Any serious damage to the film from the edge of this cut will greatly affect the resistor performance.

This particular test was repeated with the double 
cut technique (Figure 2(b)) and displayed similar drift figures.

\section{(C) Resistor Trimmed to 1,8K Ohms}

$\begin{array}{llll}\text { Q-rate } & \text { Bite size } & \text { Laser average power } & \text { Technique } \\ 5 \mathrm{KHz} & 0.002^{\prime \prime} & \mathrm{TEM}_{\text {o o }} 1.4 \text { watts } & \text { Double cut }\end{array}$

Test conditions Average post trim drift

Processing $\quad-0.05 \%$

$100 \mathrm{Hrs}$ load $\quad-0.35 \%$

$250 \mathrm{Hrsload} \quad-0.35 \%$

These resistors dropped in value after $100 \mathrm{hrs}$ loading but remained stable after this initial period. The overall results from this batch were very much better than the above batches (A) and (B), thus pointing to $\mathrm{TEM}_{00}$ as the optimum cutting mode.

\section{(D) Resistor Trimmed to $3.5 \mathrm{~K}$ Ohms}

$\begin{array}{llll}\text { Q-rate } & \text { Bite size } & \text { Laser average power } & \text { Technique } \\ 2 \mathrm{KHz} & 0.001^{\prime \prime} & \text { Multimode 6 watts } & \text { Double cut }\end{array}$

The resistive paste used in these resistors was not considered a laser-compatible paste and, as can be seen, a low beam speed and extremely high power in multimode was necessary to achieve final value. The results illustrate clearly that this is not the solution to a non-compatible paste.

$\begin{array}{ll}\text { Test conditions } & \text { Average post trim drift } \\ \text { Processing } & -2.0 \% \\ \text { 100 Hrs load } & -4.7 \% \\ 250 \text { Hrs load } & -5.0 \%\end{array}$

Some resistors in the batch drifted up to $-8.0 \%$ and the lowest figure was $-3.3 \%$.

(E) A large batch of resistors was trimmed using information from the other batches as a guide.

\section{Resistors Trimmed to $1 \mathrm{~K}$ Ohms}

\begin{tabular}{|c|c|c|c|c|}
\hline Q-rate & Bite size & \multicolumn{2}{|c|}{ Laser average power } & Technique \\
\hline $6 \mathrm{KHz}$ & $0.002^{\prime \prime}$ & $\mathrm{TEM}_{00}$ & 1.1 watts & Double cut \\
\hline \multicolumn{3}{|c|}{ Test conditions } & \multicolumn{2}{|c|}{ Average post trim drift } \\
\hline \multirow{3}{*}{\multicolumn{3}{|c|}{$\begin{array}{l}100 \text { Hrs load } \\
250 \text { Hrs load } \\
1,000 \text { Hrs load } \\
\text { Temperature rapid change }\end{array}$}} & \multicolumn{2}{|l|}{$-0.05 \%$} \\
\hline & & & \multicolumn{2}{|l|}{$-0.1 \%$} \\
\hline & & & \multicolumn{2}{|l|}{$-0.2 \%$} \\
\hline \multicolumn{5}{|c|}{$\begin{array}{l}\text { Temperature rapid change } \\
\left(-55^{\circ} \mathrm{C} \text { to }+125^{\circ} \mathrm{C}\right)\end{array}$} \\
\hline \multicolumn{3}{|c|}{ Climatic sequence 21 days } & \multicolumn{2}{|c|}{$\begin{array}{l}\text { No detectable change } \\
-0.18 \%\end{array}$} \\
\hline \multicolumn{3}{|c|}{ Long term damp heat 21 days } & \multicolumn{2}{|c|}{$-0.8 \%$} \\
\hline \multicolumn{3}{|c|}{$600 \%$ overload for 5 seconds } & \multicolumn{2}{|l|}{$-0.05 \%$} \\
\hline
\end{tabular}

\section{CONCLUSION}

As these results serve to illustrate, there is only one factor to consider when laser trimming, and this is the amount of power dissipated on the substrate. This must be kept to an absolute minimum by using the laser in $\mathrm{TEM}_{00}$ at the lowest power output necessary to achieve a clean cut at the required trimming speed, not forgetting the exception in the case of low value resistor pastes. If this technique is used in conjunction with well-screened compatible pastes then there is no reason why excellent results cannot be obtained.

When air-abrasive cutting was established as a technique, it was possible to apply it rapidly to any type of material, providing elementary precautions were taken, and good electrical results were achieved in both short and long term testing.

The laser adjusting technique which uses vapourisation of the resistor material is a far more complicated process which involves testing for compatibility of each resistive material used. This should extend to individual resistor blends within a resistor material family. This is a lengthy process as it is typical to have 25 resistor blends within a material family. However, providing this is carried out effectively, the cutting technique offers many advantages for the future.

\section{REFERENCES}

1. Proc. Conf. on Hybrid Microelectronics. I.E.R.E. Conf. Proc. No. 27. (Sept. 1973.)

2. P. Moore, 'Hybrid thick film circuits in the telecommunication industry', Ref. 1, pp. 3-14 (1973).

3. B. Walton, 'Principles of thick film material formulation', Ref. 1, pp. 39-46 (1973).

4. M. V. Coleman, 'Evaluation methods for the examination of thick film materials', Ref. 1, pp. 221-238 1973.

5. L. F. Miller, 'Silver-palladium fired electrodes', Proc. I.E.E.E. Elec. Comp. Conf. pp. 52-63 (1968).

6. U. S. Patent No. 3573229.

7. I. G. Bowkley, 'Improved, glass-ceramic, thick film capacitors', Proc. Conf. on Hybrid Microelectronics, I.E.R.E. Conf. Proc. No. 27, pp. 47 :-55 (Sept. 1973).

8. R. C. Headley, M. J. Popowich, F. J. Anders, 'YAG laser trimming of thick film resistors', Proc. I.E.E.E. Elec. Comp. Conf. pp. 47-55 (1973). 

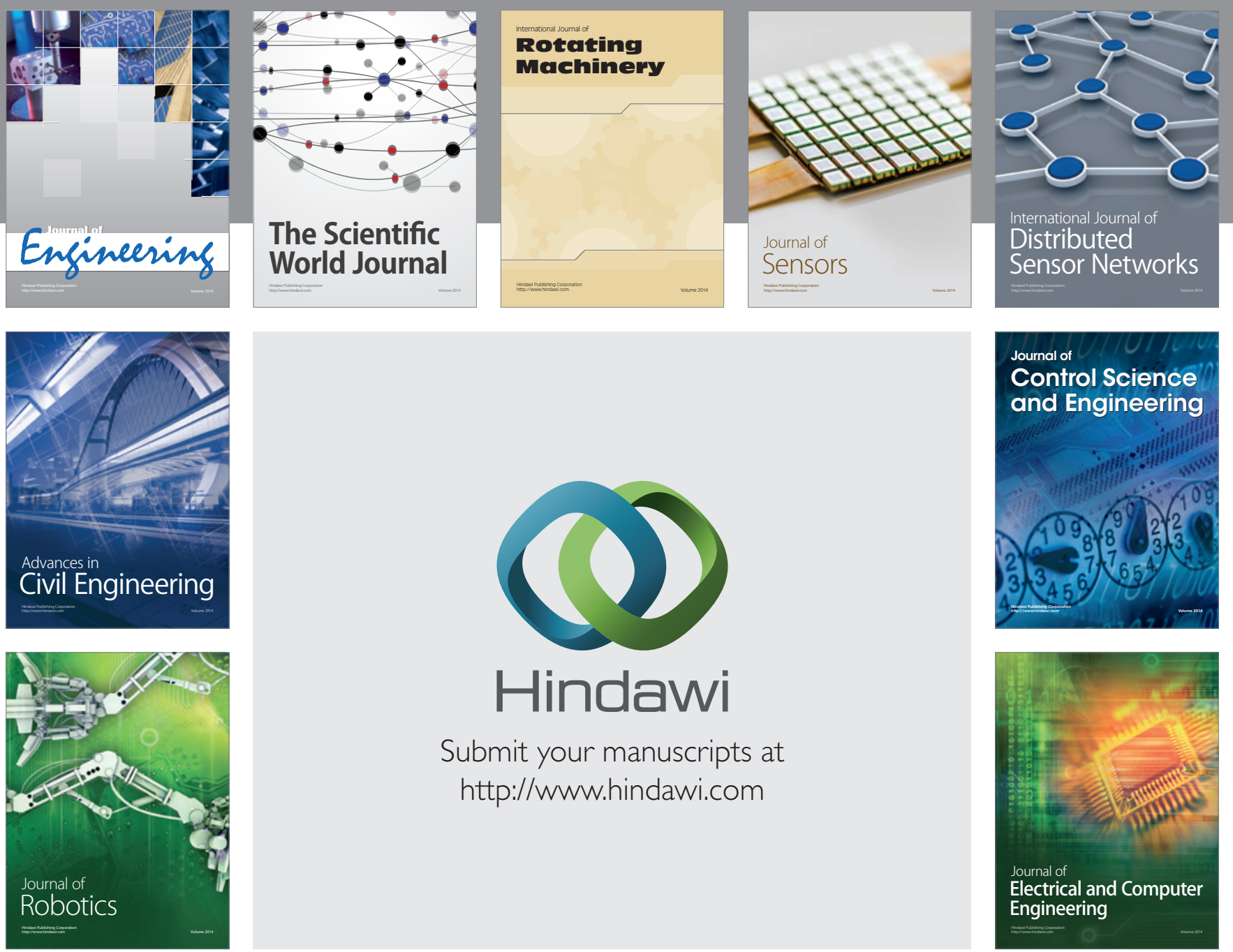

Submit your manuscripts at

http://www.hindawi.com
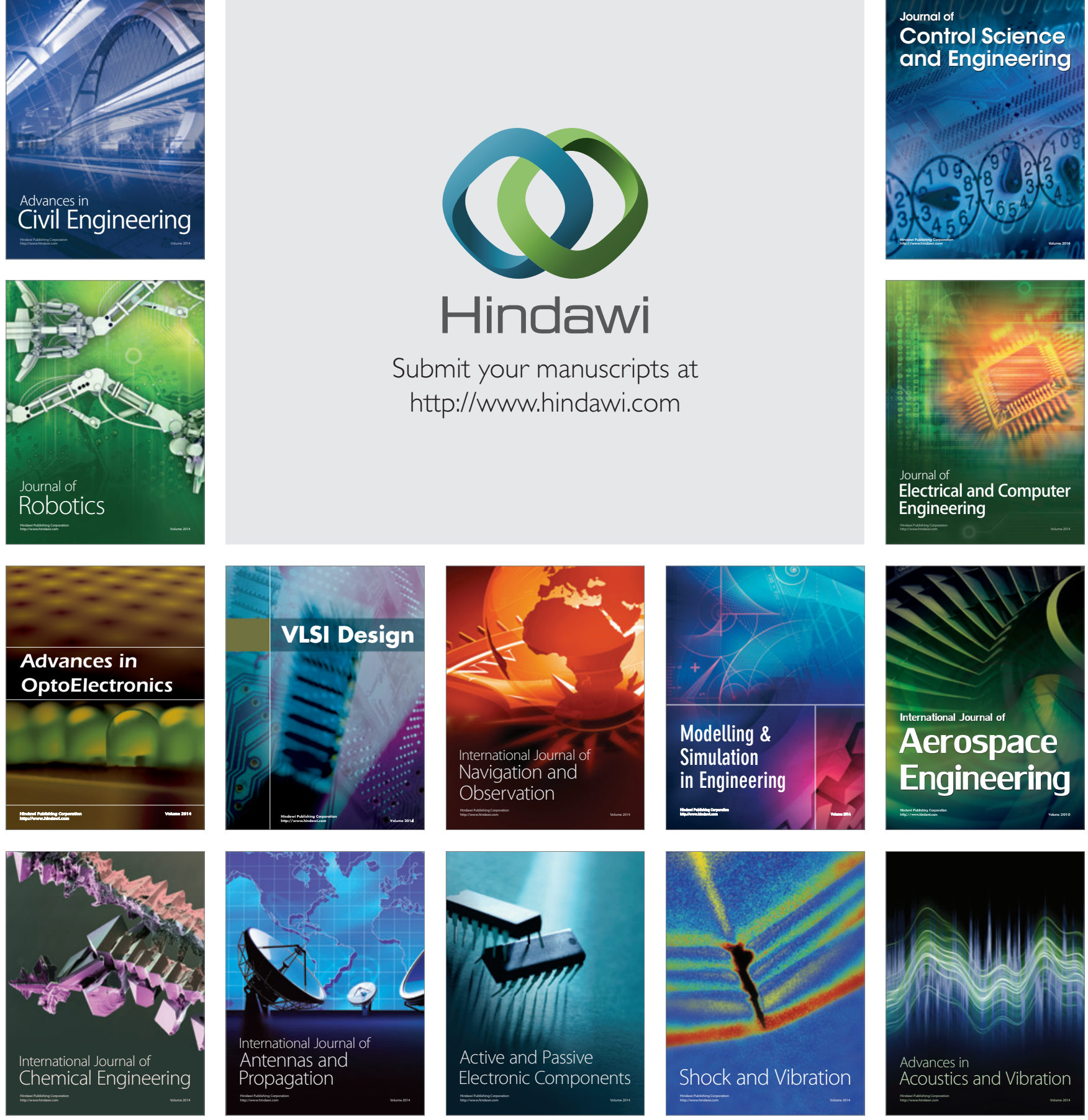